\title{
Complementary Metal Oxide Semiconductor-Compatible Top- Down Fabrication of a Ni/NiO Nanobeam Room Temperature Hydrogen Sensor Device
}

Kusuma M.B. Urs ${ }^{\dagger}$, Krutikesh Sahoo*, Navakanta Bhat ${ }^{\ddagger}, V^{\star}$ inayak Kamble ${ }^{\dagger}{ }^{*}$

${ }^{\dagger}$ School of Physics, Indian Institute of Science Education and Research Thiruvananthapuram 695551 India

Center for nano science and engineering, Indian Institute of Science Bangalore, 560012 India.

Keywords: Nanofabrication, gas sensor, NiO, hydrogen sensor, miniaturized.

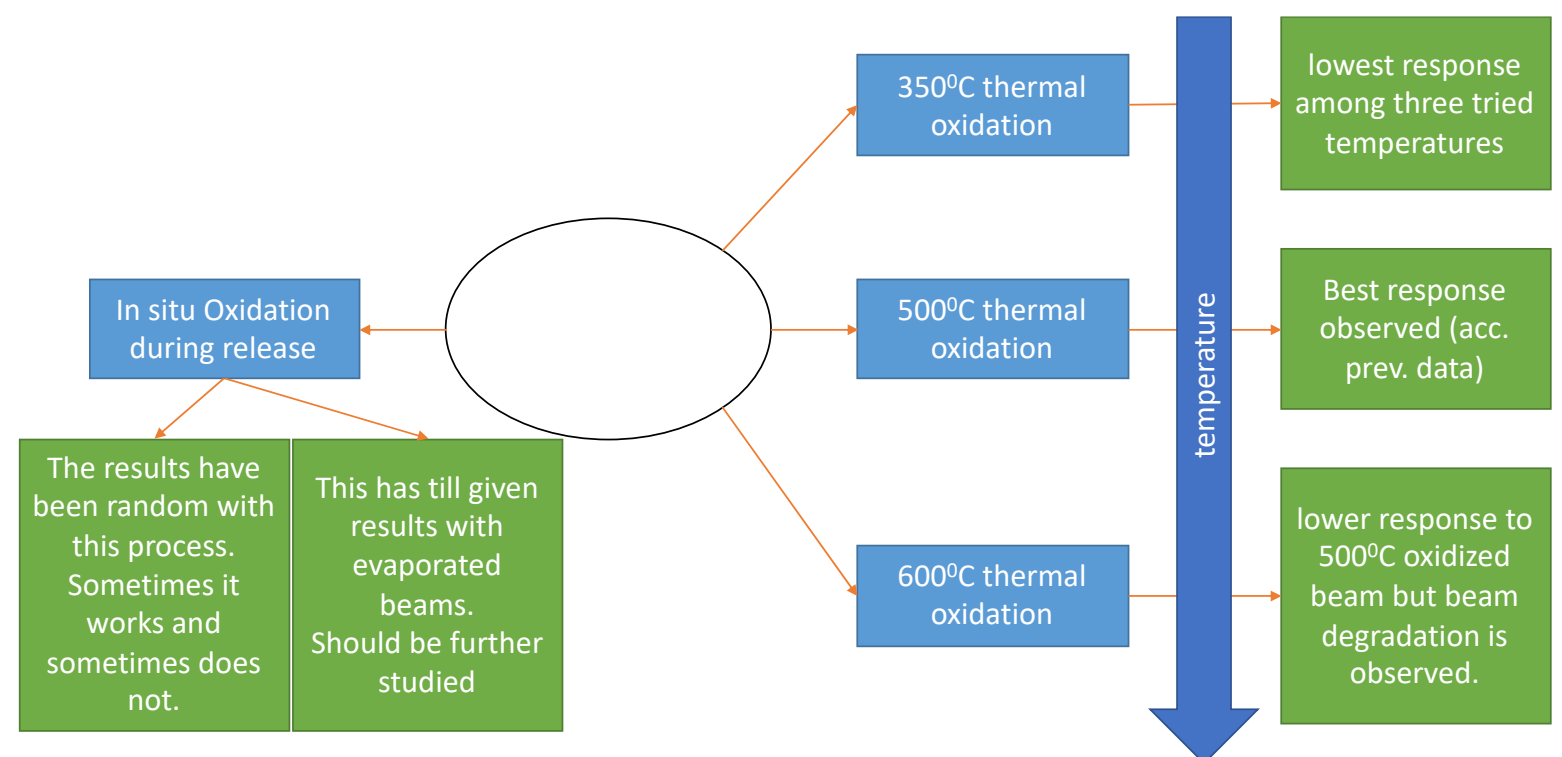

Fig S1. The graphical presentation of the empirical results observed in Fabrication of $\mathrm{Ni} / \mathrm{NiO}$ nanobeam in CMOS compatible fashion. 

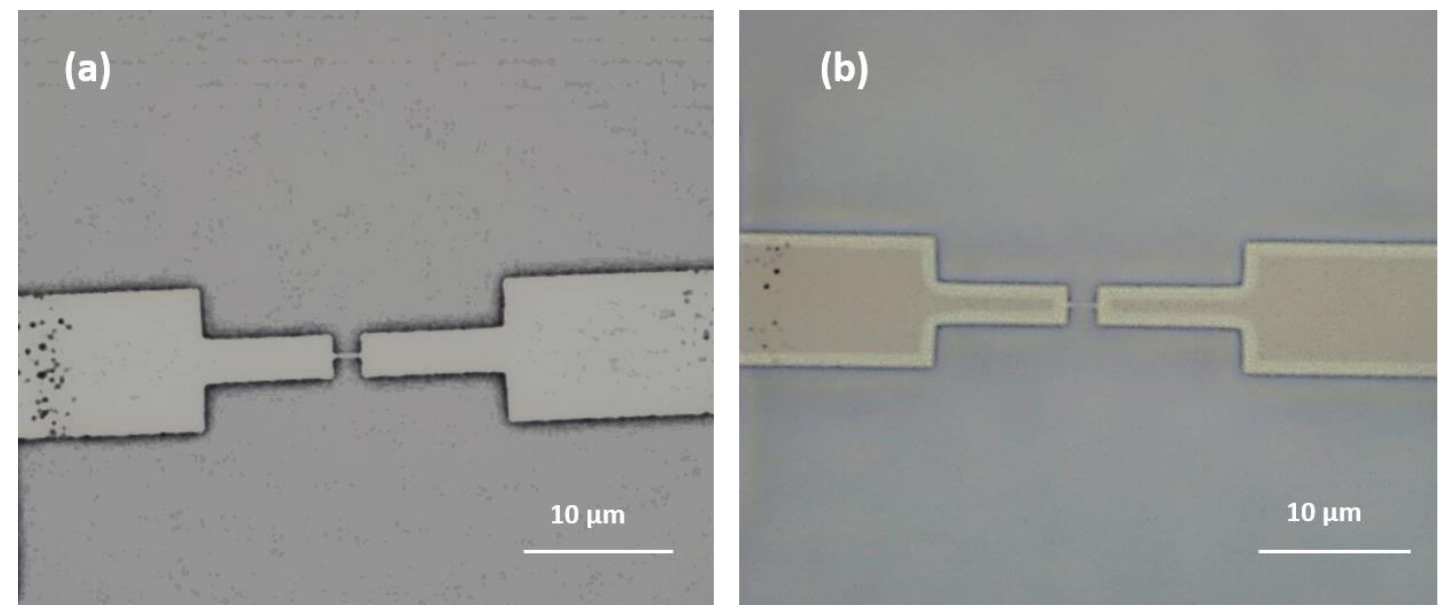

Fig S2. Optical micrograph images of the beam (a) before and (b) after oxidation

(a)

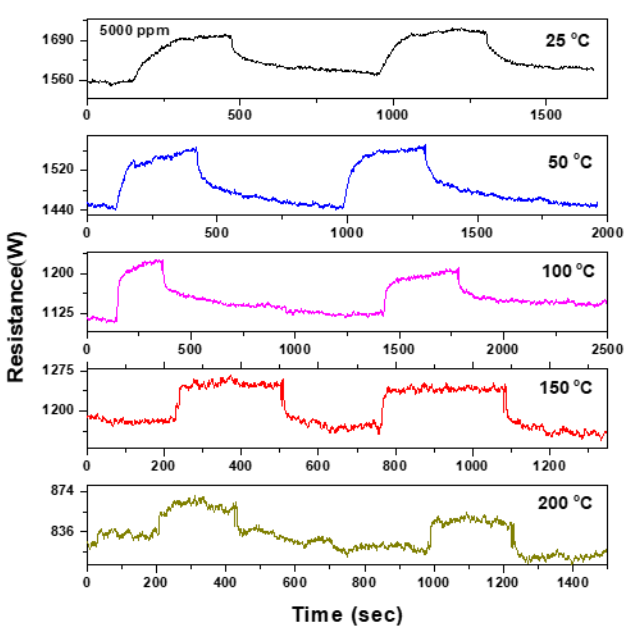

(c)

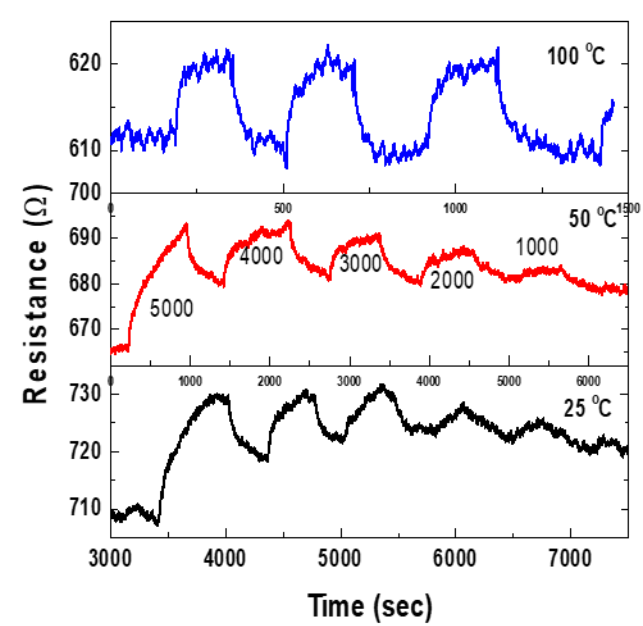

(b)

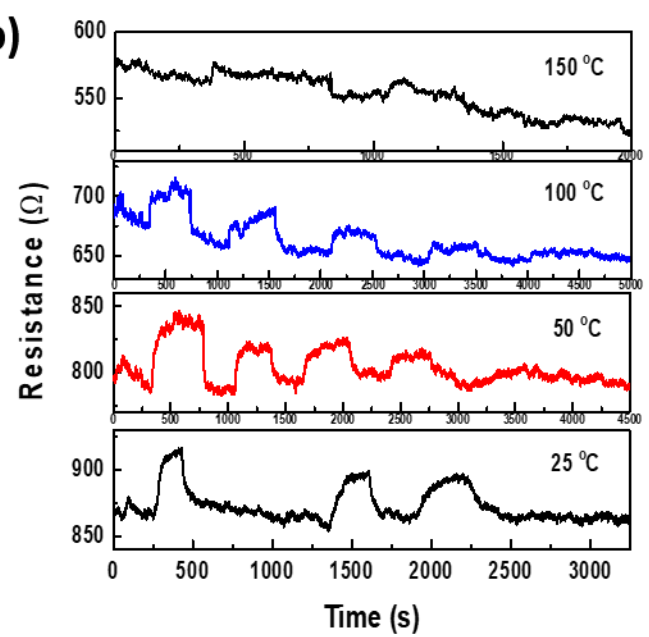

(d)

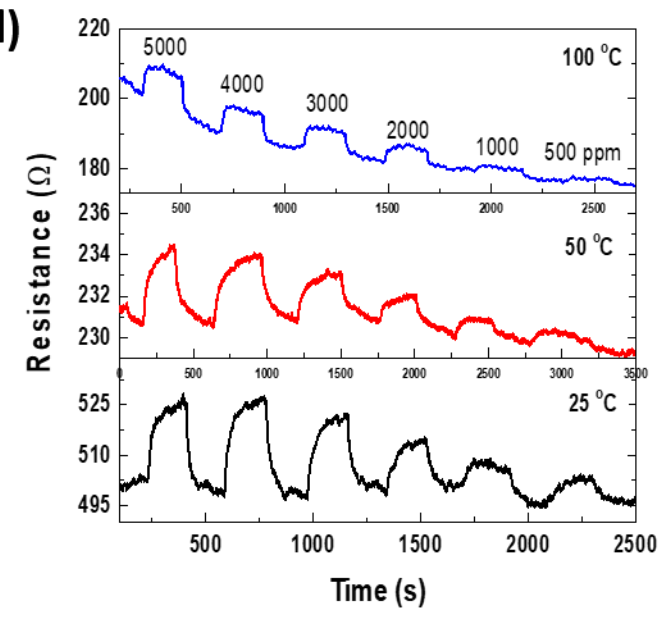

Fig S3. The transient graphs showing the sensor response for various hydrogen concentrations (a) STO $20 \mathrm{~nm}$ (b) ETO $20 \mathrm{~nm}$ (c) SPO $20 \mathrm{~nm}$ and (d) EPO $20 \mathrm{~nm}$ 

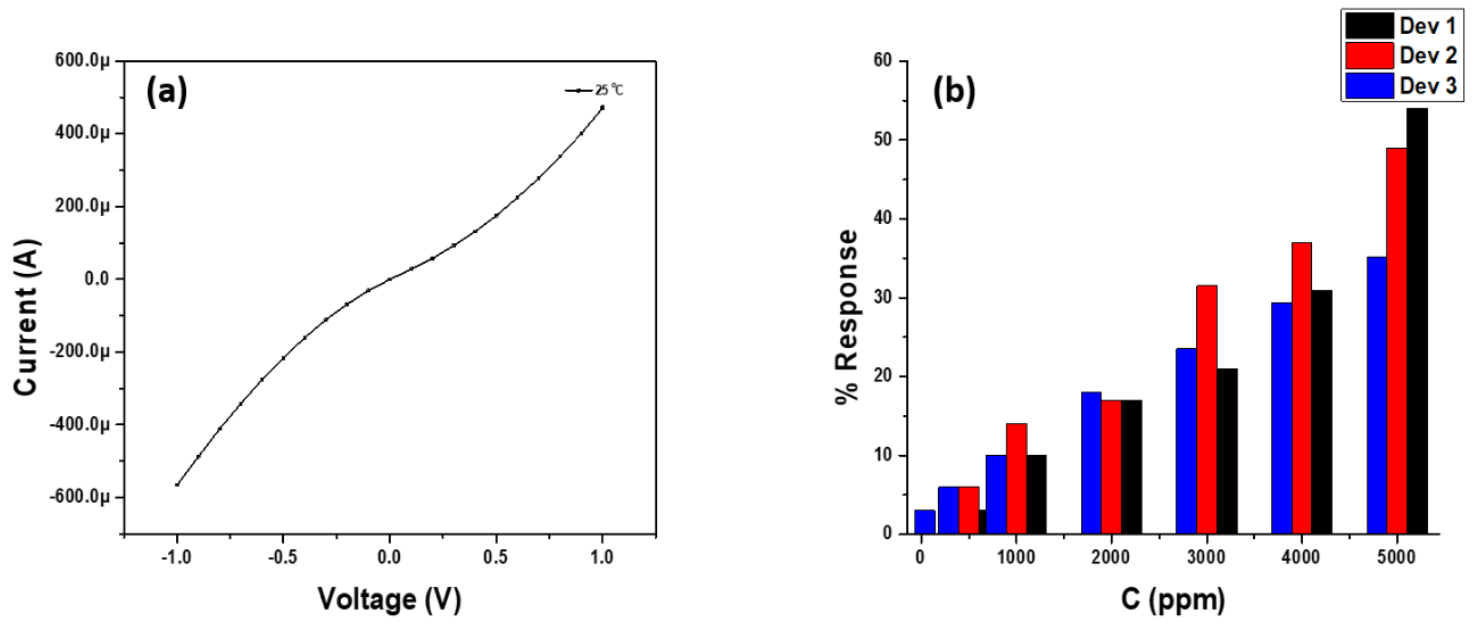

Fig S4. (a) IV characteristics of SPO $15 \mathrm{~nm}$ device and (b) the response of the $15 \mathrm{~nm}$ different devices

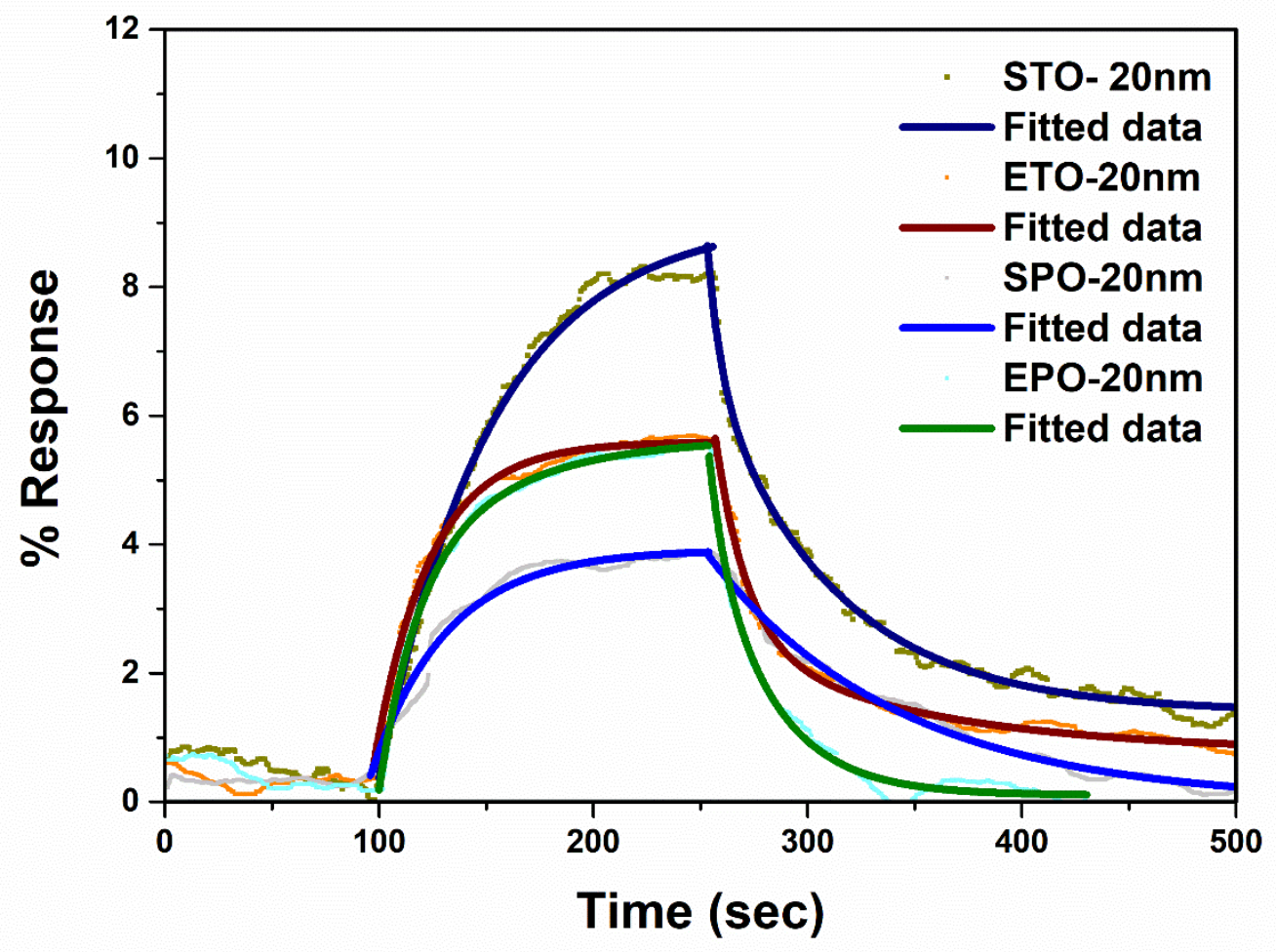

Fig S5. The typical response transients with exponential fittings to extract the time constants. 


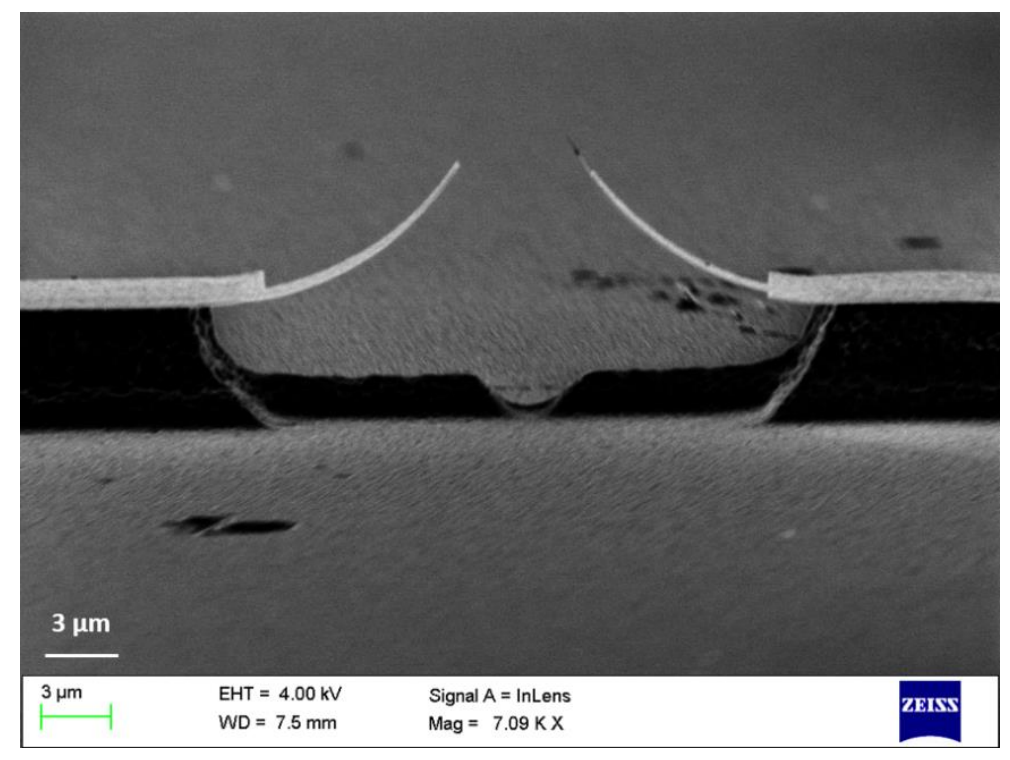

Fig S6: SEM image of the fractured beam after oxidation at $600{ }^{\circ} \mathrm{C}$ in oxygen atmosphere 